\title{
Neutrinos from TXS 0506+056 prior to the 2017 gamma-ray flare
}

\author{
Chad Finley ${ }^{1, *}$ for the IceCube Collaboration \\ ${ }^{1}$ Oskar Klein Centre, Stockholm University
}

\begin{abstract}
On 2017 September 22, IceCube released a public alert announcing the detection of a well-reconstructed, high-energy neutrino event. Such alerts issued through the Extremely High Energy (EHE) alert stream occur roughly four times a year. Subsequent multimessenger follow-up identified that the event was coincident in direction and time with a gamma-ray flare from the blazar TXS 0506+056. This association prompted an archival analysis searching for additional neutrinos from the direction of TXS 0506+056 using 9.5 years of IceCube neutrino observations. An excess of neutrino events with respect to atmospheric backgrounds was found between September 2014 and March 2015. The analysis yields $3.5 \sigma$ evidence for neutrino emission from the direction of TXS 0506+056, independent of and prior to the 2017 emission.
\end{abstract}

\section{Introduction}

The IceCube Neutrino Observatory [1] instruments one cubic kilometer of deep glacial ice at the South Pole in order to detect the Cherenkov light from relativistic charged particles created by neutrino interactions in the ice and bedrock. On 2017 September 22, a high-energy neutrino event, IceCube-170922A, was selected by the Extremely High Energy (EHE) online event filter [2], and reported as a public alert [3]. At the time, such EHE alerts were sent at a rate of about four per year, with the selection threshold set so that approximately half of the events are estimated to be astrophysical neutrinos, the rest being atmospheric background events. Further studies following the alert resulted in the best localization of the neutrino direction at equatorial coordinates RA $77.43_{-0.65}^{+0.95}$ and Dec $+5.72_{-0.30}^{+0.50}$ (degrees, J2000, 90\% containment region). The most likely neutrino energy was estimated to be $290 \mathrm{TeV}$, with a 90\% confidence level (CL) lower limit of $183 \mathrm{TeV}$ [4].

The alert led to a substantial multi-messenger follow-up campaign when it was determined by the Large Area Telescope (LAT) on the Fermi Gamma-ray Space Telescope[5] that the direction of IceCube-170922A coincided with the location of the known blazar TXS 0506+056 during a time while it was in a state of enhanced gamma-ray activity [6]. After the neutrino event, the Major Atmospheric Gamma Imaging Cherenkov (MAGIC) Telescopes were able to detect gamma rays with energies up to $400 \mathrm{GeV}$ from the blazar [7, 8]. The significance of the spatial and temporal coincidence of the high-energy neutrino and the blazar flare is estimated to be at the $3 \sigma$ level [4]. More details are given in these proceedings in Franckowiak et al. Based on this result, an analysis of archival neutrino data was

\footnotetext{
*e-mail: cfinley@fysik.su.se
} 
undertaken [9], in order to determine whether there was any evidence of previous emission of neutrinos from TXS 0506+056.

\section{Method}

The archival analysis was performed using standard maximum likelihood techniques employed in previous IceCube searches. The time-dependent analysis described in [10] searches for a temporary excess of neutrinos (above the background expectation from atmospheric neutrinos) during any time window of any duration, from seconds to years. Four parameters are fit: the number of excess events $n_{s}$, the spectral index $\gamma$ (related to the energy of the events), the central time $T_{0}$ and the duration $T_{\mathrm{W}}$ of the time window when the excess is found. As explained in [10], a "penalty" term is included in the likelihood that corrects for the fact that significant excesses are more easily found by chance during short time windows, since there are more independent opportunities ("trials") of short duration than of long duration. This factor is always included in the results below. Additionally, because the archival data consists of six separate periods (due to different stages of detector construction and data-taking procedures), a similar correction is applied in the end to arrive at the final significance. The time window is incorporated into the likelihood as a probability distribution function (PDF), effectively weighting the events according to their arrival times with respect to the PDF. The search was performed using two generic time window profiles: a Gaussian PDF and a box PDF.

\section{Results}

The search for a previous episode of neutrino emission from the direction of TXS 0506+056 was performed separately on each of the six archival data periods. The results are shown in Fig. 1. A significant excess is identified by both the Gaussian and box time windows in the fifth data period, centered in December 2014 and spanning several months. The best-fit parameters are given in table 1 . The excess consists of $13 \pm 5$ events above the expectation from the atmospheric background. The significance depends not only on the number of events reconstructed close to the coordinates of TXS 0506+056, but also the energy of the events. This is illustrated in Fig. 2.

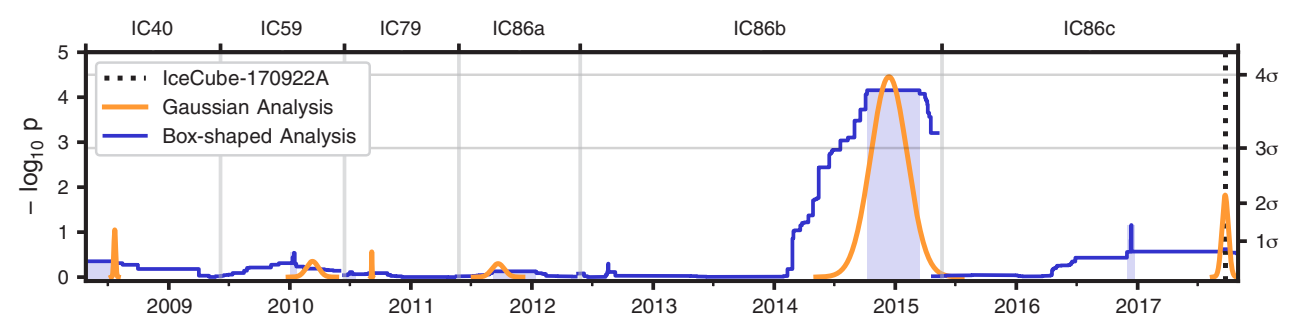

Figure 1. Best-fit time-windows and their significances in each of the six data-taking periods. In orange is the result using the Gaussian time-window PDF, with the most significant neutrino excess found in 2014/15. The blue shaded boxes are the most significant time-windows using the box PDF, with the most significant result similar to that found with the Gaussian time-window. Additionally the blue line indicates the significance of the best-fitting box time windows for other times besides the most significant window. The vertical dotted line in the last data period indicates the time of the IceCube170922A event. Figure from [9]. 


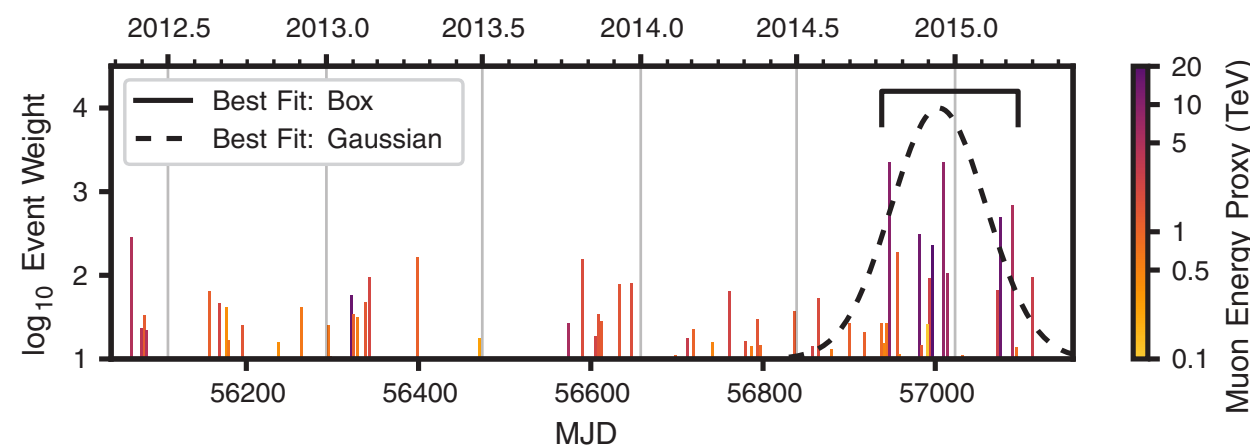

Figure 2. Time-independent weights of individual events during the 2012-2015 data-taking period. The weight is the product of the spatial and energy terms in the likelihood, where each term is given by the ratio of the signal and background PDFs evaluated for the given event. The spatial term increases depending on how close the event is reconstructed to the coordinates of TXS 0506+056 and the energy term increases with higher values of the reconstructed energy of the event. Additionally, the reconstructed muon energy is indicated by the color for each event. The dashed curve and solid brace show the most significant Gaussian and box time windows, respectively. Outside of this region, the distribution of event times and weights is compatible with background expectations. Figure from [9].

Table 1. Best-fit parameter values for the neutrino excess in 2014/15 using the Gaussian-shaped time window and the box-shaped time window.

\begin{tabular}{c|cc}
\hline Parameter & Gaussian & Box \\
\hline Central Date & 2014 Dec. 13 & 2014 Dec. 26 \\
Width & 110 days $(-1 \sigma$ to $+1 \sigma)$ & 158 days \\
$v_{\mu}+\bar{v}_{\mu}$ fluence & $2.1 \times 10^{-4} \mathrm{TeV} \mathrm{cm}^{-2}$ & $2.2 \times 10^{-4} \mathrm{TeV} \mathrm{cm}^{-2}$ \\
spectral index & 2.1 & 2.2 \\
\hline
\end{tabular}

The final significance of the neutrino excess in 2014/15 is determined as follows. First, the analysis is repeated on randomized data sets (constructed by scrambling the times of all events in the 2012-2015 data period and re-calculating the right ascension coordinates). The fraction of randomized data sets in which a more significant excess is found than in the real data is $3 \times 10^{-5}$ for the Gaussian time window, and $7 \times 10^{-5}$ for the box time window. An additional correction, due to the fact that the different data periods provide six opportunities to find a significant excess by chance, is a factor of 9.5 years / 3 years, leading to p-values of $10^{-4}$ and $2 \times 10^{-4}$, respectively. Finally, a conservative trial factor of 2 is applied to the most significant result, because the two different time window profiles provide separate (although not entirely uncorrelated) occasions to find a significant excess by chance. The final p-value is thus $2 \times 10^{-4}$, corresponding to $3.5 \sigma$.

\section{Discussion}

In standard models of neutrino production related to cosmic ray acceleration, protons interact either with photons or other protons near the source to create charged pions, which decay to neutrinos and leptons. These interactions also produce neutral pions, which decay to gamma rays. The connection between neutrinos and gamma-rays detected at Earth is not straightforward, however, because high-energy gamma rays can interact en route, either in 
the source environment if it is sufficiently dense or via pair production on background light while traversing intergalactic space. This complication notwithstanding, it is still useful to compare the neutrino and gamma-ray fluxes.

From the best-fit parameters for the muon-neutrino fluence and spectral index (table 1), we can estimate the neutrino energy fluence integrated between $32 \mathrm{TeV}$ and $3.6 \mathrm{PeV}$, which is the $68 \%$ central energy range of IceCube sensitivity for a source with an $E^{-2.1}$ spectrum at the declination of TXS 0506+056. We also assume that the muon-neutrino fluence is one-third of the total neutrino fluence. We then find an all-flavor neutrino energy fluence of $\left(4.2_{-1.4}^{+2.0}\right) \times 10^{-3} \mathrm{erg} \mathrm{cm}^{-2}$ over this energy range. The redshift of TXS 0506+056 was measured to be $z=0.3365 \pm 0.0010$ in [11], from which we can calculate an isotropic neutrino luminosity of $\left(1.2_{-0.4}^{+0.6}\right) \times 10^{47} \mathrm{erg} \mathrm{s}^{-1}$ averaged over 158 days.

In [4], the isotropic gamma-ray luminosity between $0.1 \mathrm{GeV}$ and $100 \mathrm{GeV}$ was found to be $1.3 \times 10^{47} \mathrm{erg} \mathrm{s}^{-1}$ in the \pm 2 weeks around IceCube-170922A when the blazar was in an active state. The luminosity averaged over all Fermi-LAT observations is $0.3 \times 10^{47}$ $\mathrm{erg} \mathrm{s}^{-1}$. The neutrino luminosity during the 2014/15 neutrino excess is thus of comparable magnitude to the gamma-ray luminosity during the 2017 flare, but a factor four above the long-term average, which is more representative of the gamma-ray luminosity during the period of the neutrino excess. The fact that the observed neutrino luminosity is higher than the gamma-ray luminosity during the 2014/15 neutrino excess could imply that the corresponding gamma rays expected from neutral pion decays are either absorbed, cascade down to lower energies, or arrive outside the Fermi-LAT energy band. Comprehensive multi-wavelength coverage comparable to that achieved after the IceCube-170922A event in 2017 would have been valuable during 2014/15 for better understanding. In the near future IceCube is initiating a public alert system that will be sensitive to the gradual accumulation of a neutrino excess over periods of days to weeks, which will make it possible to achieve this coverage for a similar occurrence in the future.

\section{References}

[1] M.G. Aartsen, M. Ackermann, J. Adams, J.A. Aguilar, M. Ahlers, M. Ahrens, D. Altmann, K. Andeen, T. Anderson, I. Ansseau et al., Journal of Instrumentation 12, P03012 (2017), 1612.05093

[2] M.G. Aartsen et al. (IceCube), Astroparticle Physics 92, 30 (2017), 1612.06028

[3] IceCube Collaboration, GRB Coordinates Network, Circular Service 21916 (2017)

[4] M.G. Aartsen et al. (IceCube, Fermi-LAT, MAGIC, AGILE, ASAS-SN, HAWC, H.E.S.S., INTEGRAL, Kanata, Kiso, Kapteyn, Liverpool Telescope, Subaru, Swift NuSTAR, VERITAS, VLA/17B-403), Science 361, eaat1378 (2018), 1807.08816

[5] W.B. Atwood, A.A. Abdo, M. Ackermann, W. Althouse, B. Anderson, M. Axelsson, L. Baldini, J. Ballet, D.L. Band, G. Barbiellini et al., Astrophysical Journal 697, 1071 (2009), 0902. 1089

[6] Y. T. Tanaka, S. Buson, D. Kocevski, The Astronomer's Telegram 10791 (2017)

[7] J. Aleksić, E.A. Alvarez, L.A. Antonelli, P. Antoranz, M. Asensio, M. Backes, J.A. Barrio, D. Bastieri, J. Becerra González, W. Bednarek et al., Astroparticle Physics 35, 435 (2012), 1108. 1477

[8] R. Mirzoyan, The Astronomer's Telegram 10817 (2017)

[9] M.G. Aartsen et al. (IceCube), Science 361, 147 (2018), 1807.08794

[10] J. Braun, M. Baker, J. Dumm, C. Finley, A. Karle, T. Montaruli, Astroparticle Physics 33, 175 (2010), 0912 . 1572

[11] S. Paiano, R. Falomo, A. Treves, R. Scarpa, Astrophys. J. 854, L32 (2018), 1802 . 01939 